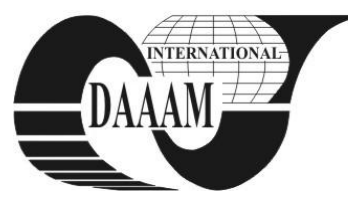

Annals of DAAAM for 2011 \& Proceedings of the 22nd International DAAAM Symposium, Volume 22, No. 1, ISSN 1726-9679 ISBN 978-3-901509-83-4, Editor B. Katalinic, Published by DAAAM International, Vienna, Austria, EU, 2011 Make Harmony between Technology and Nature, and Your Mind will Fly Free as a Bird Annals \& Proceedings of DAAAM International 2011

\title{
MULTI ROBOT PATH PLANNING FRAMEWORK
}

\author{
KESKIN, O[nur]; CETIN, L[event]; BASER, O[zgun] \& UYAR, E[rol]
}

\begin{abstract}
In this paper we present a framework for path planning and path finding for multiple mobile robots with global vision. Our framework model considers the agents' dynamic status and their environment with obstacles to perform given tasks. The global vision system provides feedback to main controller computer and mobile robots are directed towards to their tasks with avoiding obstacles and without any collision. Different kinds of scenario are prepared to simulate manipulating tasks and non-collision behavior with our framework. Experiment results with Lego Mindstorms NXT shows that our framework can be used where a multi robot system is needed with minimum resources.
\end{abstract}

Key words: multi-agent systems, mobile robot, global vision, path finding, path planning

\section{INTRODUCTION}

Multi agent system design has been developing since 1980s (Asama et. al., 1989; Fukuda \& Nakagawa, 1987). In our research topic, "agent" can be defined as a robot system that observers its environment with the help of various sensors and perform action with different types of actuator to achieve assigned task. Multi Robot Systems have received increased interest in recent years because of their remarkable properties and advantages such as fault tolerance, larger range of task domains, robustness, improved system performance and lower economic cost (Vlassis, 2007). Especially interest of multi robot system research is in developing path finding and planning, traffic control, formation control, localization, exploration and foraging. These subjects also studied in the past with single robot systems. But the concept of using multiple robots has many advantages. Multi Robot coordination has the potential to complete the task more efficiently than a single robot. Each mobile robot in Multi Robot Systems can complete different tasks in the same time by working separately which is more reasonable and suitable for the agent (Liu \& Wu, 2000).

Most of the multi robot system has to solve the shortest path problem to achieve given task. In the last decades path planning and path finding computation methods are discussed, most of new method and algorithm is published (Geramifard et. al., 2006; Tavakoli et. al., 2008).

We develop robust, reliable, programmer friendly agent based framework which uses global vision as a location feedback sensor setup, and assigns various kind of task to multiple robot in the vision space. The first experiment is to perform a task with only an agent to represent basic capabilities. The second experiment is to show how to handle with complex tasks with multiple agents. Eventually experiment results are discussed and we concluded paper with future works.

\section{PROJECT DESCRIPTION}

Our framework is design to solve guidance problem of multi mobile robots. This problem has three general modules to discuss. These modules are global vision, path finding and vehicle control (Stone,2007).

First part is gathering data from environment with only a single camera. The reasons for this usage are to decrease the cost of total system and moreover to share all the data between robots. It is better to manage them from one centralized computer. Centralized computing and managing unit can foresee actions and behave considering the whole working environment. We can express this choice with an everyday example. In a laboratory, students can be distributed into some number of groups or individually to make the experiment, ask their own questions for their own problems to the teacher. Or whole students can make the experiments under teacher's surveillance. This approach comes with a great advantage. The students do not only learn their own problems' solutions, they can learn, or access all the solutions in the laboratory class. And hiring one teacher for each student is never considered as a reasonable option because of cost and redundancy. So we use this principle to supply feedback from our mobile robots with the help of global vision system.

Second part is to finding the shortest path from initial location of a mobile robot to its target location. This step is also combining two sub modules; one of them is used for tracking each mobile robot by the help of global vision system. Second sub module is called "collision checker" which plans the motion step of each mobile robot.

And the last module is used for sending driving parameters and reading mobile robot device information from each mobile robot.

\section{DEMONSTRATION}

We run two different experiments in order to observe how our guidance control framework solves problems in these experiments (figure 1). First experiment is done for how simply framework can be use by programmers. Our framework can also be used from graphical user interface (Çok Silahşorlar,2009).

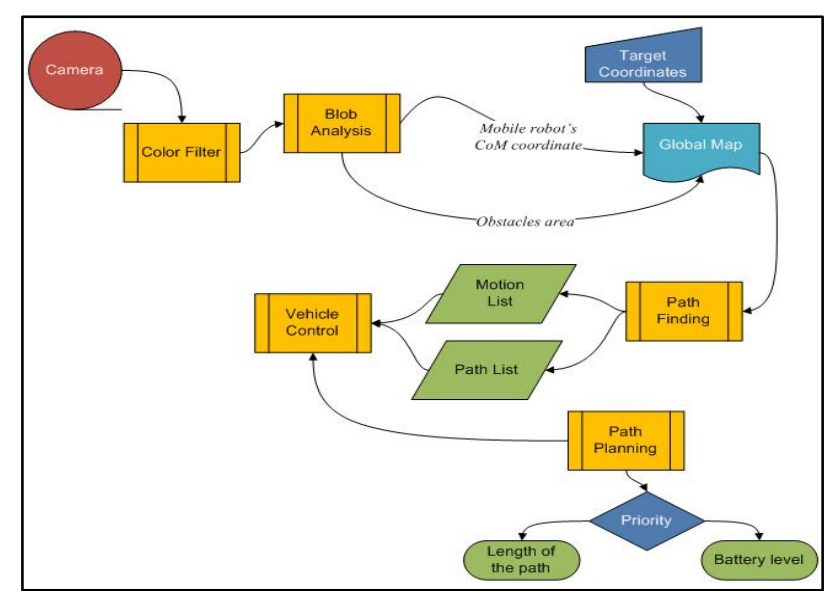

Fig. 1. Multi robot guidance control flow diagram 


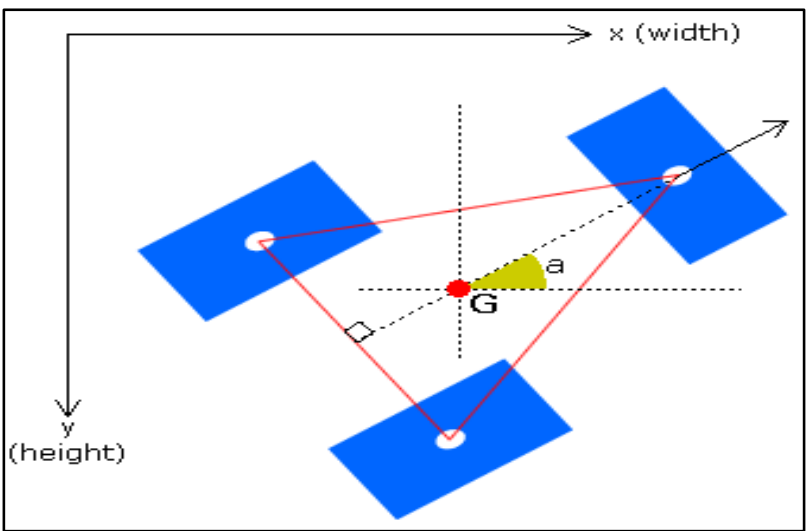

Fig. 2. Mobile vehicle's marker for blob analysis, "G" for position, "a" angle for rotation of a mobile robot

In graphical user interface (figure 3), the first step is to select connected capture device from camera list, then connect each mobile robot by selecting virtual communication port from COM port list, after this mobile robots' name, and battery information are taken if suitable ports are given. If user wants to set a virtual mobile robot, it can be manually added to the system.

After the connection procedure, vehicle drive module start to blob analysis, until computer vision module finishes calculating mobile robots' coordinate according to labels COG. With the help of these labels, framework can easy knows mobile robots both coordinates in the global map and orientation. Framework also knows which communication port control which mobile robot, each mobile robot's name, battery level, identification pattern color, and current coordinate. The next step is to generate global map. This is computed by computer vision module, and obstacles are found by using connected components labeling. The next step is to assign task, for experiment it will be "going from A to B with shortest path". For this step path finding module computes shortest map according to global map and target coordinate for each mobile robot. When proper paths are found, priorities sorts which mobile robot is continue when paths are crossed. Then framework is ready to start the task for all system. When the task continues, mobile robots are tracked by computer vision module with the help of identification color pattern. The tracking can be very complex when the mobile robot population is large. User can change the sample time for tracking or it can also leave as real-time tracking.

Second experiment will demonstrate how multi robots can avoid randomly moving obstacles while running their own tasks. Dynamic obstacles can be a human, or for a multi robot task, the other robots are the obstacle to each other. Computer vision module and path planning module work cooperatively to avoid and generate new path for each mobile robot. In the planning procedure priorities of each agent is considered.

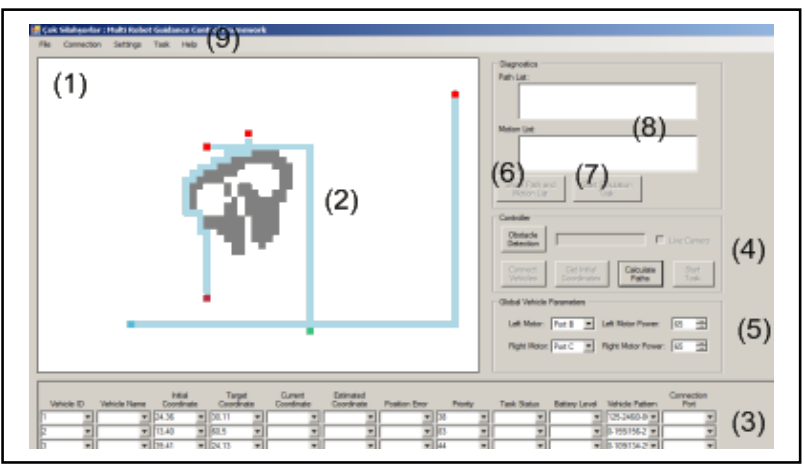

Fig. 3. Screenshot of framework's GUI with mobile robots 1 -Live camera and path controller. 2-Mobile robots and their paths. 3-Mobile robot property controller grid. 4-Camera and vehicle connection, obstacle selection, vehicle recognition, path calculation and task starting buttons. 5-Global vehicle parameters. 6-Path and motion list calculation control for selected mobile robot. 7-Simulation control of the defined system. 8-Path and motion list for selected mobile robot. 9-Standard controller menu for all parameters and help section.

\section{CONCLUSION}

In this paper, a multi robot path planning framework is defined and explained how it is developed and control via framework's own graphical user interface. Framework has design to be robust, reliable and programmer-friendly. We successfully control our Lego NXT mobile robots for given different tasks. We simulate virtual mobile robots for what-if scenarios by entering their coordinates, battery level. Battery levels can assign as a priority option. With the help of this option, mobile robot behaviors and success rates can be examine without setting up the real system in the field. And if the system fails from any given scenario, system does not cause any damage on any mobile robots in reality. After simulation task, real time task is executed. Different static obstacles and dynamic obstacles are placed in the environment. As a dynamic obstacle, the other mobile robots are automaticly assigned via computer vision module. Framework can drive mobile robots via vehicle drive module smoothly different than see on figure 6 (Ahmadzadeh et. al.,2007).

As a feature work, computer vision module is going to recognize 2D barcodes (e.g. QRCode) for tracking and identification. This will be main consideration because in industrial applications color based tracking and identification cannot be accurate for mobile robots due to environmental conditions.

\section{REFERENCES}

Ahmadzadeh, A.; Jadbabaie, A., Kumar, V., Pappas, G.J. (2007). Stable Multi-Particle Systems and Application in Multi-Vehicle Path Planning and Coverage. In: 46th IEEE Conference on Decision and Control, New Orleans

Asama, H.; Matsumoto, A. \& Ishida, Y. (1989). Design of an autonomous and distributed robot system: ACTRESS. In Proceedings of the IEEE/RSJ International Workshop on Intelligent Robots and Systems, pages 283-290, Tsukuba

Geramifard, A.; Chubak, P. \& Bulitko, V. (2006). Biased Cost Path finding. Proceedings of the Artificial Intelligence and Interactive Digital Entertainment Conference (AIIDE).page 112-114, California

Fukuda, T.; Nakagawa, S. (1987). A dynamically reconfigurable robotic system. In Proceedings of the International Conference on Industrial Electronics, Control, and Instrumentation, pages 588-595, Cambridge, MA

Liu, J.; Wu, J. (2000). Multi-Agent Robotic Systems. CRC, Florida

Stone, P. (2007). Intelligent Autonomous Robotics: A Robot Soccer Case Study. Morgan \& Claypool

Tavakoli, Y.; Javadi, H.H.S. \& Adabi, S. (2008). A Cellular Automata Based Algorithm for Path Planning in MultiAgent Systems with A Common Goal. IJCSNS International Journal of Computer Science and Network Security

Vlassis, N. (2007). A Concise Introduction to Multi-Agent Systems and Distributed Artificial Intelligence. Morgan \& Claypool

*** (2009) http://code.google.com/p/coksilahsorlar "Çok Silahşorlar": A framework for multi mobile robot guidance control, Accessed on: 2011-02-01 